•研究报告・

\title{
基于功能性状的水杉原生母树种群生境适应策略
}

\author{
陈 俊 姚 兰 ${ }^{*}$ 艾训儒 朱 江 吴漫玲 \\ 黄小陈思艺王进朱强 \\ (湖北民族大学林学园艺学院, 湖北恩施 445000)
}

摘要: 植物的功能性状变异和表型可塑性是其应对异质生境的主要机制，对植物的生长和分布有重要贡献。本文 以湖北星斗山国家级自然保护区的水杉(Metasequoia glyptostroboides)原生母树为研究对象, 分析了母树种群功能 性状对树木形态、地形因子及人为干扰的响应机制。结果表明: 水杉原生母树叶面积、叶干重和比叶面积的变异 幅度大, 可塑性较强, 而枝和叶的干物质含量稳定性最高。人为干扰和 4 个地形因子均对每个功能性状变异方差有 5\%-20\%的解释度，冠幅对枝、叶干物质含量的变异方差有高达 $38 \%$ 和 $76 \%$ 的解释度。 5 个功能性状主要受海拔、 坡位和人为干扰影响, 其中, 比叶面积对环境因子和干扰的响应规律不明显, 叶面积和叶干重在强烈人为干扰的 环境中普遍增大, 枝和叶的干物质含量对坡向的变化最敏感。总之, 水杉原生母树种群通过功能性状变异对环境 能产生一定的可塑性响应, 但人为干扰对母树生长影响较大, 建议人工辅助更新, 并适度减少农业和建筑对现存 母树的影响。

关键词: 水杉; 功能性状; 种内变异; 表型可塑性; 适应策略

\section{Adaptive strategies of functional traits of Metasequoia glyptostroboides parent trees to changing habitats}

Jun Chen, Lan Yao*, Xunru Ai, Jiang Zhu, Manling Wu, Xiao Huang, Siyi Chen, Jin Wang, Qiang Zhu School of Forestry and Horticulture, Hubei Minzu University, Enshi, Hubei 445000

\begin{abstract}
Functional trait variability and phenotypic plasticity are the main mechanisms plants use to respond to heterogeneous habitats. These can determine how well a plant grows and where it is distributed. In the Xingdoushan National Nature Reserve, we assessed the response of the functional traits of a population of Metasequoia glyptostroboides parent trees to tree morphology, terrain factors and human disturbance. We found that the leaf area (LA), leaf dry weight (LDW) and specific leaf area (SLA) had large variation and great plasticity, while leaf dry matter content (LDMC) and twig dry matter content (TDMC) were more stable. Human disturbance and the four terrain factors together explained $5 \%-20 \%$ of variance for each functional trait, and crown size explained 38\% and 76\% of the variation in TDMC and LDMC, respectively. The five functional traits were mainly affected by altitude, slope aspect and human disturbance. The SLA responded slightly to environmental factors and disturbance pattern, while LA and LDW generally increased with strong disturbance. LDMC and TDMC were most sensitive to change in slope aspect. Taken together, the population of $M$. glyptostroboides parent trees demonstrated significant plasticity in response to the environment through its variability in functional traits. Because human disturbance had a great influence on the growth of these trees, artificial regeneration is recommended, and the impact of agriculture and human construction needs to be reduced.
\end{abstract}

Key words: Metasequoia glyptostroboides; functional trait; intraspecific variability; phenotypic plasticity; adaptive strategy

收稿日期: 2019-03-24; 接受日期: 2019-05-16

基金项目: 国家重点研发计划(2016YFC0503100) “极小种群野生植物种质资源保护技术研究与示范”课题(2016YFC0503102)

* 通讯作者 Author for correspondence. E-mail: hbmyyl@163.com 
植物功能性状(plant functional trait)指影响植物 存活、生长、繁殖速率和最终适合度的生物特征, 同 时是植物在漫长的进化过程中, 采用特定生态策略 或属性适应周围环境的结果(Cornelissen et al, 2003; 刘晓娟和马克平, 2015)。植物功能性状的种内变异 表征了植物沿生物和非生物梯度上的最大适应能 力, 从根本上决定着植物的生态位宽度(Ackerly \& Cornwell, 2007; Albert et al, 2010)。同时, 种内变异 可使物种通过非生物和生物篮选过程进入局域群 落(Jung \& Muller，2010)。如Laforest-Lapointe等 (2014)发现种内性状变异是决定植物能否应对气候 变化和其他环境因子改变的重要因素。因此, 种内 变异有助于解释环境梯度上种群的结构与动态。

基于植物功能性状的种群生境适应策略近年 来受到广泛关注，学者们发现物种对环境的适应性 进化所导致的种内变异对种群和群落构建机制的 影响不容忽视(Auger \& Shipley, 2013; 陈否等, 2014; Siefert et al, 2015; 唐青青等, 2016)。异质生境中, 植物的不同功能性状之间存在权衡, 通过表型可塑 性达到对生境的适应是植物的一种重要生态对策 (Gao et al, 2013; 李西良等, 2014)。植物表型可塑性 指个体在响应环境过程中改变表型的能力, 有助于 植物在异质生境中最大限度地获取资源, 进行资源 的再分配，从而实现资源的有效利用，提高植物适 合度(施建敏，2014; 王姝和周道玮，2017)。如罗琦 等(2018)通过测定光合、水力等功能性状的变化, 分 析了木麻黄(Casuarina equisetifolia)、椰子(Cocos nucifera) 等5 种植物的环境适应性, 以进行植被恢复 评价。

水杉(Metasequoia glyptostroboides)是中国著名 的杉科子遗植物, 其原生母树被列为国家一级保护 植物, 在世界植物学界有“活化石”之称, 已列入世 界自然保护联盟(IUCN) 2013年濒危物种红色名录。 目前, 湖北星斗山国家级自然保护区内的5,661株 水杉原生母树种群趋于衰退, 幼龄个体严重缺乏 (林勇等, 2017)。同时, 只有现存的水杉原生母树种 群保留着其最完整的基因库 ${ }^{1}$ 。因此, 本研究基于每 株水杉原生母树个体的形态、地形和人为干扰数据, 通过大规模的功能性状采样, 分析该种群功能性状 的结构和可塑性, 以及对地形和人为干扰的响应,

(1) 崔敏燕 (2011) 濒危物种水杉种群的引种和生存力分析. 硕士学位 论文, 华东师范大学, 上海.
旨在探讨该种群的生境适应策略, 为进一步开展水 杉原生母树的保护和恢复工作提供科学依据。

\section{材料与方法}

\section{1 研究对象}

水杉原生母树主要分布在湖北省恩施市星斗 山国家级自然保护区西部小河片区 $\left(108^{\circ} 31^{\prime}-\right.$ $108^{\circ} 48^{\prime}$ E、 $\left.30^{\circ} 04^{\prime}-30^{\circ} 14^{\prime} \mathrm{N}\right)$, 分布面积约 $60,000 \mathrm{ha}$, 涉及 45 个行政村。种群所处的自然环境和生态条件 较差, 50.89\%的个体分布在居民房前屋后(间距小于 $30 \mathrm{~m})$, 因房屋建筑、道路维修、作物种植等, 种群 受到严重的人为干扰(林勇等, 2017)。

\section{2 研究方法}

\subsection{1 水杉原生母树种群调查}

2013年7月至2014年8月，根据相关资料对现存 5,661株挂牌的水杉原生母树进行了详细调查, 主 要包括：(1)母树个体测树因子检测，包括胸径 $(\mathrm{DBH}) 、$ 树高 $(\mathrm{H}) 、$ 冠幅(crown size)和GPS坐标; (2) 地形因子调查, 包括坡度、坡位、坡向和海拔 (852-1,565 m); (3)人为干扰程度调查, 根据母树个 体生长位置(房前屋后、道路、河流、水田、苗戋、 山坡和森林)及植被状况, 采用强烈干扰、中度干 扰、轻微干扰进行分级。

\subsection{2 功能性状的测定}

2017年7-9月，根据水杉原生母树种群区域分 布、海拔梯度和胸径分布特征, 篮选出 1,208 株个体 进行植物功能性状调查。参考Pérez-Harguindeguy 等(2013)的功能手册指南, 每株个体采集4片完整、 无病虫害的健康成熟叶片，以及2段2-3年生枝条， 每个枝条长约 $5 \mathrm{~cm}$, 直径约 $1 \mathrm{~cm}$, 用自封袋密封并 放入保鲜箱。枝叶样本在 $12 \mathrm{~h}$ 内处理, 用电子天平 称取鲜重, 叶片用A4扫描仪(CanoScan LiDE 120)结 合Image J软件测量叶面积。然后, 将新鲜叶片和去 皮的枝条烘干至恒重，用电子天平测量叶片和枝条 的干重。选择叶干重(leaf dry weight, LDW)、叶面 积(leaf area, LA)、比叶面积(specific leaf area, SLA)、 叶干物质含量(leaf dry matter content, LDMC)、枝干 物质含量(twig dry matter content, TDMC) 5 个指标 作为功能性状指标。

\section{3 数据处理}

采用多元因子分析 (multiple factor analysis, MFA)检测水杉原生母树 5 个功能性状指标、形态特 
征(胸径、树高和冠幅)、地形因子(海拔、坡向、坡 度和坡位)及人为干扰强度共 13 个因子之间的相关 关系; 运用 $\mathrm{R}$ 程序包 “nlme”中的限制最大似然法, 选择广义线性模型和 “varcomp” 函数对功能性状在 形态、地形和人为干扰角度的变异进行方差分解; 采用灰色关联度分析地形因子对功能性状的影响; 采用偏相关分析(partial correlation)结合单因素方差 分析(one-way ANOVA)和Tukey HSD Test事后比较 分析, 分别检测功能性状指标针对海拔、坡向、人 为干扰的响应。数据分析参照Borcard等(2011)总结 的数量生态学方法, 在 R 3.4.4中完成。显著度设定 为 $P<0.05$ 。

\section{2 结果}

\section{1 功能性状的分布模式}

水杉原生母树种群的叶干重为单峰偏左分布, 偏度和峰度远大于其他功能性状(表1), 且统计分析 发现叶干重有较多的极小值, 其极大值远大于正常 水平。由表1可知, 5 个功能性状指标的变异幅度为 9.059\%-37.172\%, 平均变异系数为 $23.420 \%$, 叶干 重、叶面积和比叶面积可塑性较强, 而枝的干物质 含量可塑性最低。

\section{2 功能性状、形态及地形因子的相关性}

多元因子分析仅保留相关关系最为显著的因 子, 胸径、冠幅、海拔因子与功能性状指标相关性 最强, 海拔是地形因子和人为干扰因子中的主导因子 (图1)。叶干重、比叶面积、叶干物质含量与水杉原 生母树的胸径、冠幅相关, 同时受到海拔因子的强 烈影响。叶面积、枝干物质含量与树木形态、地形因 子和人为干扰的相关性较弱, 具有较强的稳定性。

\section{3 不同因子对功能性状影响的方差分解}

功能性状变异方差分解的结果见图2。人为干 扰和地形因子(海拔、坡位、坡向、坡度)对5个功能
性状变异方差的影响规律较为一致, 且均对叶干物 质含量的解释度最小; 胸径和树高对功能性状变异 方差的解释非常有限，仅胸径对枝干物质含量有 $17 \%$ 的解释，其余的解释度小于 $5 \%$; 冠幅对枝、叶 干物质含量分别有高达 $38 \%$ 和 $76 \%$ 的解释度，但对 叶面积、比叶面积和叶干重的解释度几乎为零。

\section{4 功能性状与地形因子灰色关联度分析}

选取海拔、坡向、坡度和坡位 4 个典型的地形 因子，分析功能性状受地形因子影响程度的相对大 小(表2)。叶干重、比叶面积、叶干物质含量的影响 因子大小均表现为: 海拔 > 坡向 > 坡度 > 坡位, 叶面积、枝干物质含量的影响因子大小表现为: 海 拔 > 坡度 > 坡向 > 坡位, 说明地形因子中海拔 对 5 个功能性状的影响程度最大，坡位的影响力 最小，坡向对叶干重、比叶面积和叶干物质含量的 影响较大, 坡度主要影响叶面积和枝干物质含量 指标。

\section{5 功能性状值在坡向梯度上的变化}

从图3可以看出，在从阴坡、平地到阳坡的坡向 梯度变化中, 叶干重、比叶面积呈波动变化, 无明 显规律。平地的水杉原生母树叶面积普遍较大，与 半阴坡和半阳坡差异显著 $(P$ 值分别为 0.001 和 0.037$)$, 同时阴坡和阳坡的叶面积差异不大 $(P=0.898)$ 。叶 干物质含量在半阴坡处普遍较低，且有更多的极小 值，与阴坡、平地差异极显著 $(P$ 值分别为 0.003 和 0.001)。枝干物质含量在阴坡、半阴坡和平地梯度 上变化平稳, 在半阳坡和阳坡明显上升。

\section{6 不同人为干扰程度下功能性状的分布}

偏相关分析发现，剔除地形因子的干扰后，叶 干重、叶面积、比叶面积、叶干物质含量共 4 个叶 性状与人为干扰相关性较强, 而枝干物质含量与人 为干扰不相关。因此，用单因素方差分析检测人为 干扰对 4 个叶性状的影响(图4)。由图4可知, 叶干重

表1 水杉原生母树功能性状及其变异

Table 1 Functional trait and its variance of Metasequoia glyptostroboides parent trees

\begin{tabular}{|c|c|c|c|c|c|c|c|}
\hline \multirow{2}{*}{$\begin{array}{l}\text { 性状 } \\
\text { Trait }\end{array}$} & \multicolumn{2}{|c|}{ 平均值 \pm 标准差 最小值 } & \multirow{2}{*}{$\begin{array}{l}\text { 最大值 } \\
\text { Maximum }\end{array}$} & \multirow{2}{*}{$\begin{array}{l}\text { 中值 } \\
\text { Median }\end{array}$} & \multirow{2}{*}{$\begin{array}{l}\text { 偏度 } \\
\text { Sewness }\end{array}$} & \multirow{2}{*}{$\begin{array}{l}\text { 峰度 } \\
\text { Kurtosis }\end{array}$} & \multirow{2}{*}{$\begin{array}{l}\text { 变异系数 } \\
\text { Coefficient of } \\
\text { variation (\%) }\end{array}$} \\
\hline & Mean \pm SD & Minimum & & & & & \\
\hline 叶干重 Leaf dry weight (g) & $0.229 \pm 0.085$ & 0.096 & 0.889 & 0.210 & $17,406.545$ & 11.361 & 37.172 \\
\hline 叶面积 Leaf area $\left(\mathrm{cm}^{2}\right)$ & $28.316 \pm 5.813$ & 5.419 & 61.215 & 28.903 & $6,389.909$ & 2.264 & 20.389 \\
\hline 比叶面积 Specific leaf area $\left(\mathrm{cm}^{2} / \mathrm{g}\right)$ & $11.6 \pm 3.735$ & 1.915 & 32.454 & 11.322 & $4,838.621$ & 0.983 & 32.121 \\
\hline 叶干物质含量 Leaf dry matter content (g/g) & $0.242 \pm 0.045$ & 0.129 & 0.430 & 0.237 & $4,740.165$ & 0.902 & 18.358 \\
\hline 枝干物质含量 Twig dry matter content (g/g) & $0.415 \pm 0.038$ & 0.283 & 0.618 & 0.415 & $5,497.453$ & 1.527 & 9.059 \\
\hline
\end{tabular}




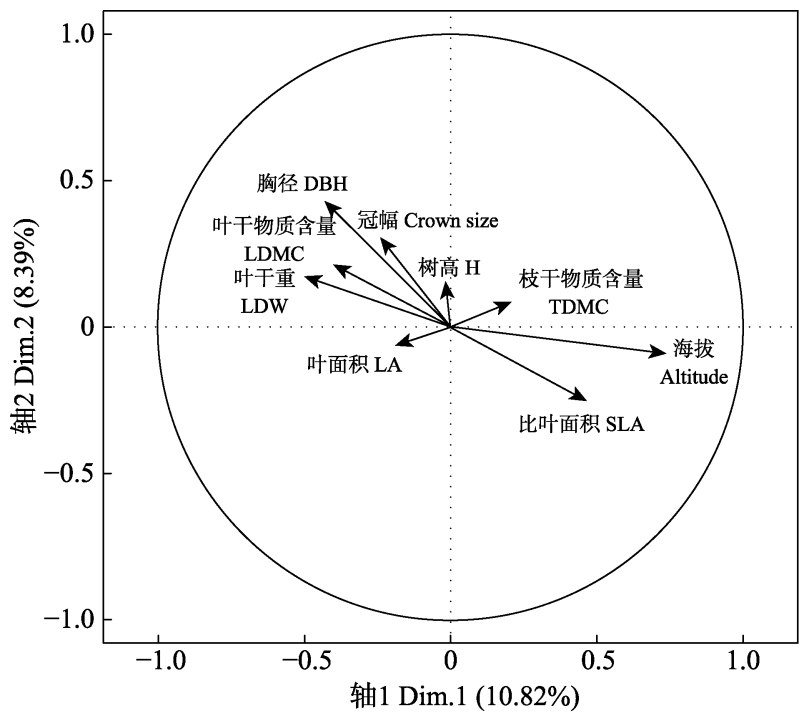

图1 功能性状、形态因子与地形因子的多元因子分析 Fig. 1 Multiple factor analysis of functional traits, tree forms, and topographical factors. LA, Leaf area; SLA, Specific leaf area; LDW, Leaf dry weight; LDMC, Leaf dry matter content; TDMC, Twig dry matter content; DBH, Diameter at breast height; H, Height.
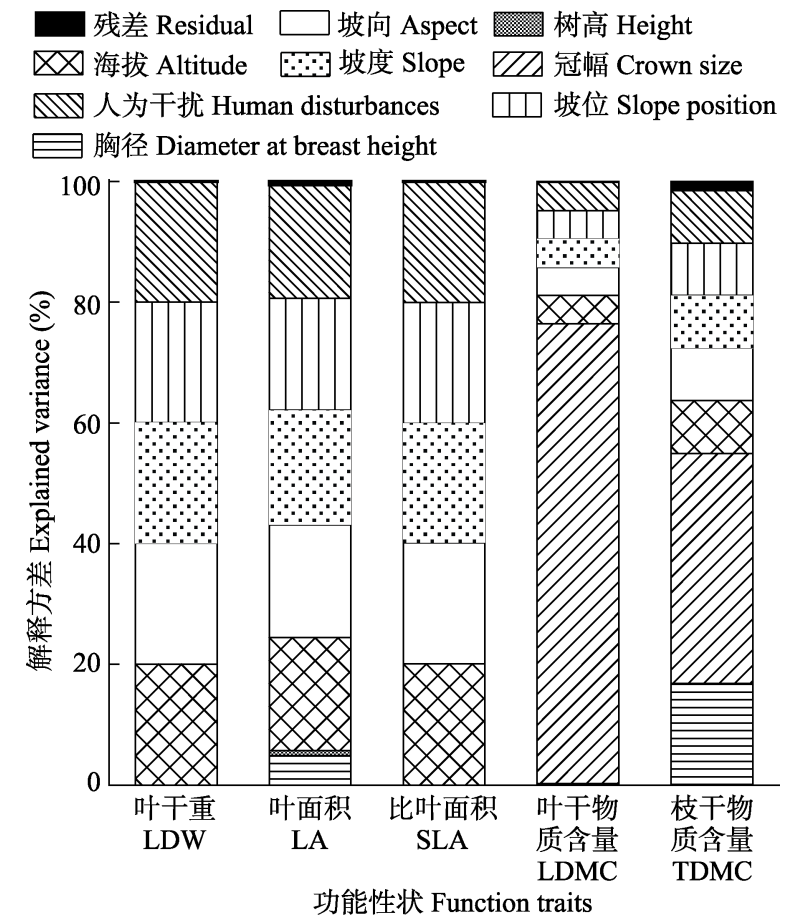

图2 不同因子对功能性状影响的方差分解

Fig. 2 Variance partitioning for function traits by different factors. LA, Leaf area; SLA, Specific leaf area; LDW, Leaf dry weight; LDMC, Leaf dry matter content; TDMC, Twig dry matter content.

在强烈干扰的环境中均值和极差较大, 变化幅度大, 同时与中度干扰差异极显著 $(P=0.004)$; 叶面积总 体呈平稳上升趋势, 且随着人为干扰变强, 其极差
表2 功能性状与地形因子灰色关联度分析

Table 2 Grey relational grade analysis between functional traits and topographic factors

\begin{tabular}{lllll}
\hline $\begin{array}{l}\text { 性状 } \\
\text { Trait }\end{array}$ & $\begin{array}{l}\text { 海拔 } \\
\text { Altitude }\end{array}$ & $\begin{array}{l}\text { 坡向 } \\
\text { Aspect }\end{array}$ & $\begin{array}{l}\text { 坡度 } \\
\text { Slope }\end{array}$ & $\begin{array}{l}\text { 坡位 } \\
\text { Slope } \\
\text { position }\end{array}$ \\
\hline 叶干重 Leaf dry weight & 0.788 & 0.724 & 0.653 & 0.542 \\
叶面积 Leaf area & 0.719 & 0.620 & 0.632 & 0.499 \\
$\begin{array}{l}\text { 比叶面积 Specific leaf area } \\
\text { 叶干物质含量 }\end{array}$ & 0.875 & 0.774 & 0.762 & 0.601 \\
Leaf dry matter content & 0.786 & 0.616 & 0.606 & 0.506 \\
$\begin{array}{l}\text { 枝干物质含量 } \\
\text { Twig dry matter content }\end{array}$ & 0.770 & 0.567 & 0.626 & 0.480 \\
\hline
\end{tabular}

增大, 变化幅度增大; 比叶面积和叶干物质含量 在不同人为干扰程度下呈波动变化, 差异不显著 $(P>0.05)$ 。

\section{3 讨论}

3.1 水杉原生母树功能性状种内变异与功能性状 可塑性

表型可塑性研究包含所有类型(如形态、分配、 生理、行为、物候、生活史等)的性状变化, 其中形 态性状是最为直观和广泛的植物性状之一(翟偲涵 等, 2017)。相对于叶面积、叶干物质含量和枝干物 质含量, 水杉原生母树种群叶干重和比叶面积的变 异幅度较大，且对表征温度环境的海拔因子响应强 烈，趋向于通过权衡面积、比叶面积等性状来响应 环境变化, 从而适应更加多样化的生境和占据更加 广阔的地理范围。这与前人的相关研究结果一致。 如: 段娜等(2018)通过干旱胁迫实验, 验证了欧李 (Cerasus humilis)通过调整形态特性和各器官生物 量对不同干旱胁迫条件产生较强的可塑性。李晓玲 等(2018)在三峡库区的研究中发现, 中华蚊母树 (Distylium chinense)的叶片功能性状对环境变化较 为敏感且具有较强的可塑性, 即面对不同生境具有 较宽的生态幅。以上研究表明, 水杉原生母树叶片 功能性状变化能有效反映其表型可塑性, 是表型可 塑性研究的重要基础。

\section{2 水杉原生母树种群的生境适应策略}

植物个体会根据它们的功能性状值确定其功 能策略, 对所处环境进行响应(Kraft et al, 2015)。本 研究发现, 水杉原生母树种群的叶干重、叶面积、 叶干物质含量均与胸径呈显著正相关, 表明大树具 

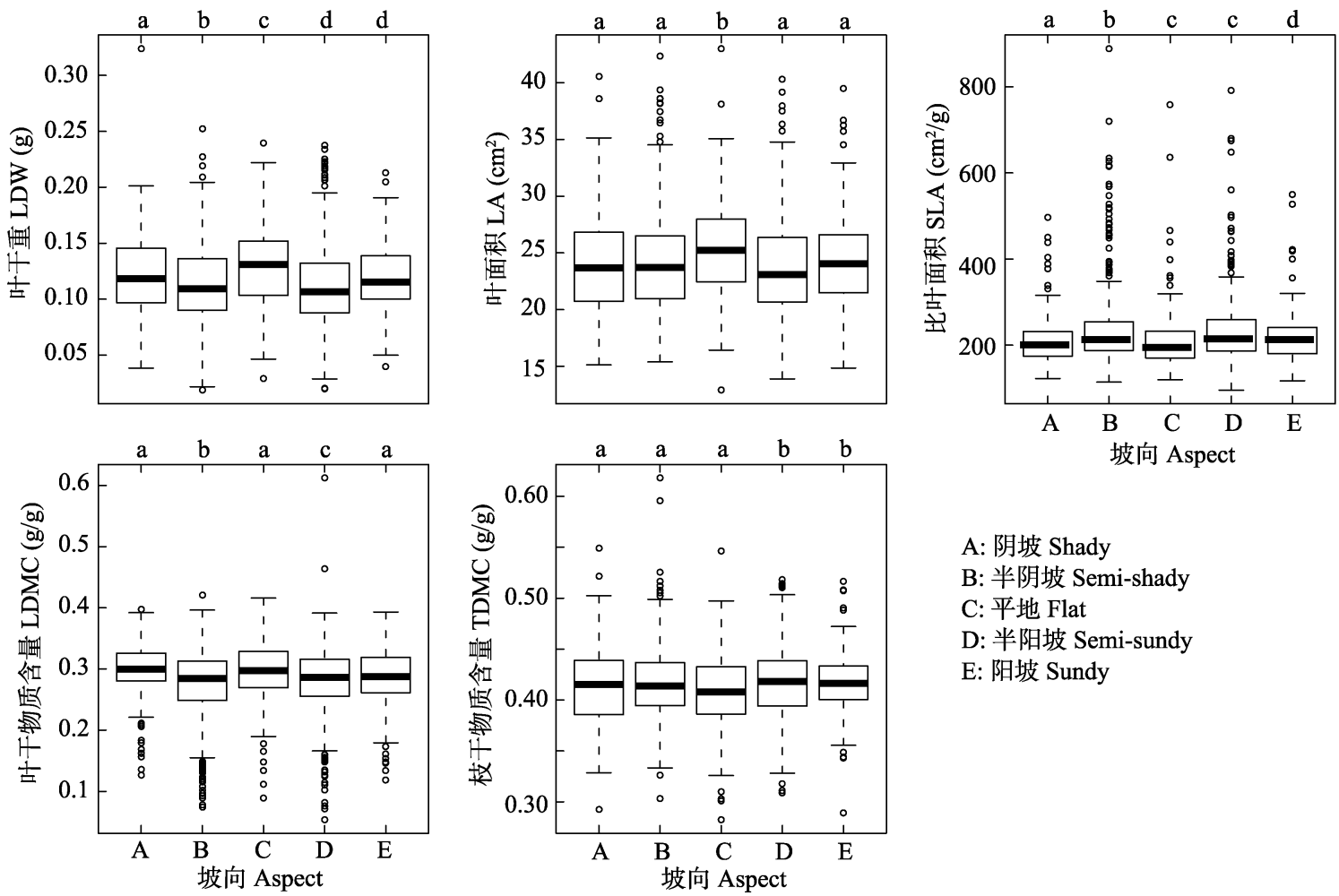
A: 阴坡 Shady
B: 半阴坡 Semi-shady
C: 平地 Flat
D: 半阳坡 Semi-sundy
E: 阳坡 Sundy

图3 功能性状值在坡向梯度上的变化

Fig. 3 Changes of functional trait values on the gradient of slope aspects. LA, Leaf area; SLA, Specific leaf area; LDW, Leaf dry weight; LDMC, Leaf dry matter content; TDMC, Twig dry matter content.
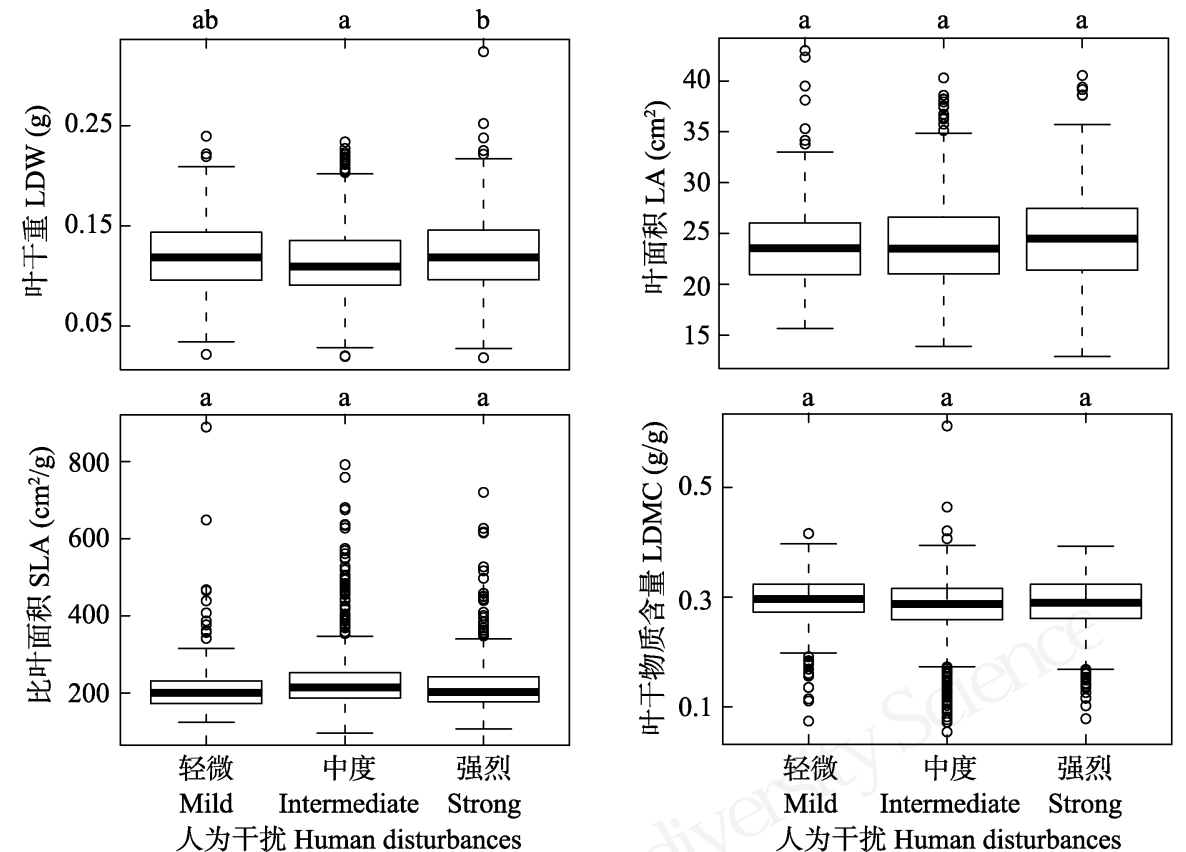

图4 人为干扰下功能性状值的分布

Fig. 4 Distribution of function trait values under human disturbances. LA, Leaf area; SLA, Specific leaf area; LDW, Leaf dry weight; LDMC, Leaf dry matter content.

有更强的捕获光能、制造和储存有机物的能力, 与 水杉速生、喜光的特性一致。熊彪等(2009)发现水
杉原生母树的树高、胸径和冠幅均与坡向因子显著 相关, 但本研究中水杉原生母树叶面积、比叶面积、 
叶干重性状对坡向因子的响应不明显。此外, 表征 植物生长速率的母树叶、枝干物质含量在半阳坡和 阳坡明显上升, 枝、叶器官生物量的积累对光照的 响应强烈, 同时, 表征对光照资源捕捉能力的比叶 面积对地形因子响应不明显, 且极低的比叶面积 (均值0.116 $\mathrm{cm}^{2} / \mathrm{g}$ ) 降低了单位叶面积的呼吸碳损失, 这表明水杉母树的叶面积、比叶面积和叶干重的可 塑性弱于树高、冠幅和胸径这3个形态因子，母树在 坡向梯度上更倾向于权衡树高、冠幅和胸径来适应 生境变化。

\section{3 水杉原生母树种群的保护建议}

水杉原生母树自20世纪40年代被发现以来一 直受到国家的重视和保护, 其中至少3,000株位于 房屋、道路、苗圃和河流附近的母树得到有效保护, 但周围的非自然环境不适合母树种子繁殖, 难以天 然更新, 同时, 图 3 中人为干扰对 5 个性状具有 5\%-20\%的解释度，也间接反映了非自然环境的影 响作用。在人为干扰强烈的环境中, 水杉原生母树 叶面积、叶干重性状值变异程度更大, 比叶面积和 枝、叶干物质含量变化不显著, 人为干扰可能对母 树叶性状已经产生影响, 而母树的枝、叶的生长速 率和资源利用状态保持稳定。此外, 建筑、道路、 苗围、耕地可能导致母树的水环境干旱、贫㾑。综 上所述，水杉原生母树种群保护工作需要考虑到不 同海拔和地形条件下性状的明显差异, 关注平地和 阴坡处母树的长势，同时注重人为干扰环境中母树 的保护，限制水杉母树周围的民房，尽量拆除猪 圈、围墙、弃房等建筑，降低人为活动对水杉原生 母树光照、水热环境的干扰。

\section{参考文献}

Ackerly DD, Cornwell WK (2007) A trait-based approach to community assembly: Partitioning of species trait values into within- and among-community components. Ecology Letters, 10, 135-145.

Albert CH, Thuiller W, Yoccoz NG, Soudant A, Boucher F, Saccone P, Lavorel S (2010) Intraspecific functional variability: Extent, structure and sources of variation. Journal of Ecology, 98, 604-613.

Auger S, Shipley B (2013) Inter-specific and intra-specific trait variation along short environmental gradients in an old-growth temperate forest. Journal of Vegetation Science, 24, 419-428.

Borcard D, Gillet F, Legendre P (2011) Numerical Ecology with R. Springer Science \& Business Media, New York.
Chen L, Mi XC, Ma KP (2014) Niche differentiation and its consequence on biodiversity maintenance in forest communities. Chinese Bulletin of Life Sciences, 26, 112-117. (in Chinese with English abstract) [陈磊, 米湘成, 马克平 (2014) 生态位分化与森林群落物种多样性维持研究展 望. 生命科学, 26, 112-117.]

Cornelissen JHC, Lavorel S, Garnier E, Díaz S, Buchmann N, Gurvich DE, Reich PB, ter Steege H, Morgan HD, van der Heijden MGA, Pausas JG, Poorter H (2003) A handbook of protocols for standardised and easy measurement of plant functional traits worldwide. Australian Journal of Botany, 51, 335-380.

Duan N, Xu J, Chen HL, Gao JL, Liu YT, Jia YK (2018) Effects of drought stress on phenotypic plasticity of Cerasus humilis. Guihaia, 39, 1159-1165. (in Chinese with English abstract) [段娜，徐军，陈海玲，高君亮，刘禹廷，贾玉奎 (2018) 干旱胁迫对欧李幼苗表型可塑性的影响. 广西植 物, 39, 1159-1165.]

Gao L, Li B, Liu WY, Shen YX, Liu WJ (2013) Inhibition effects of daughter ramets on parent of clonal plant Eichhornia crassipes. Aquatic Botany, 107, 47-53.

Jung V, Muller S (2010) Intraspecific variability and trait-based community assembly. Journal of Ecology, 98, 1134-1140.

Kraft NJB, Adler PB, Godoy O, James EC, Fuller S, Levine JM (2015) Community assembly, coexistence and the environmental filtering metaphor. Functional Ecology, 29, 592-599.

Laforest-Lapointe I, Martínez-Vilalta J, Retana J (2014) Intraspecific variability in functional traits matters: Case study of Scots pine. Oecologia, 175, 1337-1348.

Liu XJ, Ma KP (2015) Plant functional traits-Concepts, applications and future directions. Scienta Sinica Vitae, 45, 325-339. (in Chinese with English abstract) [刘晓娟，马克 平 (2015) 植物功能性状研究进展. 中国科学: 生命科 学, 45, 325-339.]

Li XL, Hou XY, Wu XH, Sarula, Ji L, Chen HJ, Liu ZY, Ding Y (2014) Plastic responses of stem and leaf functional traits in Leymus chinensis to long-term grazing in a meadow steppe. Chinese Journal of Plant Ecology, 38, 440-451. (in Chinese with English abstract) [李西良, 侯向阳, 吴新宏, 萨茹拉, 纪否, 陈海军, 刘志英, 丁勇 (2014) 草甸草原 羊草茎叶功能性状对长期过度放牧的可塑性响应. 植物 生态学报, 38, 440-451.]

Li XL, Wen HR, Wang XS, Yang J, Huang CM (2018) Phenotypic plasticity of Distylium chinense leaves in relation to soil environmental factors in heterogeneous habitats in the Three Gorges Reservoir Region. Acta Ecologica Sinica, 38, 3581-3591. (in Chinese with English abstract) [李晓玲, 温 浩然, 王雪松, 杨进, 黄成名 (2018) 三峡库区不同生境 下中华蚊母树叶片表型可塑性及其与土壤环境因子的关 系. 生态学报, 38, 3581-3591.]

Lin Y, Ai XR, Yao L, Guo QJ, Zhang MX, Chen J (2017) Population structure and dynamics of Metasequoia glyptostroboides parent trees. Chinese Journal of Ecology, 36, 
1531-1538. (in Chinese with English abstract) [林勇, 艾训 儒, 姚兰, 郭秋菊, 张敏霞, 陈俊 (2017) 水杉原生母树 种群结构与动态. 生态学杂志, 36, 1531-1538.]

Luo Q, Liu H, Wu GL, He PC, Hua L, Zhu LW, Zhang H, Liu N, Jian SG, Ye Q (2018) Using functional traits to evaluate the adaptability of five plant species on tropical coral islands. Acta Ecologica Sinica, 38, 1256-1263. (in Chinese with English abstract) [罗琦, 刘慧, 吴桂林, 贺鹏程, 华 雷, 朱丽薇, 张辉, 刘楠, 简曙光, 叶清 (2018) 基于功 能性状评价5种植物对热带珊瑚岛环境的适应性. 生态学 报, 38, 1256-1263.]

Pérez-Harguindeguy N, Díaz S, Garnier E, Lavorel S, Poorter H, Jaureguiberry P, Bret-Harte MS, Cornwell WK, Craine JM, Gurvich DE, Urcelay C, Veneklaas EJ, Reich PB, Poorter L, Wright IJ, Ray P, Enrico L, Pausas JG, de Vos AC, Buchmann N, Funes G, Quétier F, Hodgson JG, Thompson K, Morgan HD, ter Steege H, Heijden MGAVD, Sack L, Blonder B, Poschlod P, Vaieretti MV, Conti G, Staver AC, Aquino S, Cornelissen JHC (2013) New handbook for standardised measurement of plant functional traits worldwide. Australian Journal of Botany, 61, 167-234. Siefert A, Violle C, Chalmandrier L, Albert CH, Taudiere A, Fajardo A, Aarssen LW, Baraloto C, Carlucci MB, Cianciaruso MV, de L Dantas V, de Bello F, Duarte LDS, Fonseca CR, Freschet GT, Gaucherand S, Gross N, Hikosaka K, Jackson B, Jung V, Kamiyama C, Katabuchi M, Kembel SW, Kichenin E, Kraft NJB, Lagerström A, Bagousse-Pinguet YL, Li Y, Mason N, Messier J, Nakashizuka T, Overton JM, Peltzer DA, Pérez-Ramos IM, Pillar VD, Prentice HC, Richardson S, Sasaki T, Schamp BS, Schöb C, Shipley B, Sundqvist M, Sykes MT, Vandewalle M, Wardle DA (2015) A global meta-analysis of the relative extent of intraspecific trait variation in plant communities. Ecology Letters, 18, 1406-1419.
Shi JM, Ye XH, Chen FS, Yang QP, Li ZY, Fang K, Yang GY (2014) Adaptation of bamboo to heterogeneous habitat: Phenotypic plasticity. Acta Ecologica Sinica, 34, 5687-5695. (in Chinese with English abstract) [施建敏, 叶学华, 陈伏 生, 杨清培, 黎祖尧, 方楷, 杨光耀 (2014) 竹类植物对 异质生境的适应一一表型可塑性. 生态学报, 34, 5687-5695.]

Tang QQ, Huang YT, Ding Y, Zang RG (2016) Interspecific and intraspecific variation in functional traits of subtropical evergreen and deciduous broad-leaved mixed forests. Biodiversity Science, 24, 262-270. (in Chinese with English abstract) [唐青青, 黄永涛, 丁易, 藏润国 (2016) 亚热带 常绿落叶阔叶混交林植物功能性状的种间和种内变异. 生物多样性, 24, 262-270.]

Wang S, Zhou DW (2017) Research on phenotypic plasticity in plants: An overview of history, current status, and development trends. Acta Ecologica Sinica, 37, 8161-8169. (in Chinese with English abstract) [王姝, 周道玮 (2017) 植物 表型可塑性研究进展. 生态学报, 37, 8161-8169.]

Xiong B, Yao L, Yi YM, Wang BQ, Fan SH (2009) Research on growth of the Metasequoia glyptostroboides mother trees. Journal of Hubei University for Nationalities (Natural Science Edition), 27, 439-442. (in Chinese with English abstract) [熊彪, 姚兰, 易咏梅, 王柏泉, 范深厚 (2009) 水杉原生母树生长势调查研究. 湖北民族学院学报(自然 科学版), 27, 439-442.]

Zhai SH, Wang P, Sheng LX (2017) Phenotypic plasticity of plant functional traits in competition environments. Journal of Beihua University (Natural Science), 18, 538-546. (in Chinese with English abstract) [翟偲涵, 王平, 盛连喜 (2017) 竞争条件下植物功能性状的表型可塑性研究进 展. 北华大学学报(自然科学版), 18, 538-546.]

(责任编委: 藏润国 责任编辑: 黄祥忠) 